\title{
COMMENTARY
}

\section{Smoking and autoimmune thyroid disease: the plot thickens}

\author{
Gerasimos E Krassas* and Wilmar Wiersinga ${ }^{1, *}$ \\ Department of Endocrinology, Diabetes and Metabolism, Panagia General Hospital, Tsimiski 92, Thessaloniki 54622, Greece and ${ }^{1}$ Department of \\ Endocrinology and Metabolism, Academic Medical Center, University of Amsterdam, The Netherlands
}

(Correspondence should be addressed to G E Krassas; Email: krassas@the.forthnet.gr)

*Authors contributed equally to this study

\begin{abstract}
New studies have shown that smoking may protect against the development of thyroid peroxidase antibodies, which may result in a decreased risk of Hashimoto's hypothyroidism (HH), whereas it stimulates the development of Graves' hyperthyroidism (GH). According to the above-mentioned hypothesis, to stop smoking would decrease the risk of GH but increase the risk of $\mathrm{HH}$. Also, smoking has been identified as one of the risk factors for the development or worsening of eye changes after ${ }^{131} \mathrm{I}$ treatment of GH. Additionally, the outcome of medical treatment of Graves' ophthalmopathy (GO) is less favourable in smokers as compared to non-smokers. There is concern also about the effect of passive smoking on autoimmune thyroid disease. In a recent study it has been shown that the latter may have a deleterious effect on childhood GO.
\end{abstract}

European Journal of Endocrinology 154 777-780

The association between smoking and autoimmune thyroid disease (AITD), as evident from epidemiological studies, has become common knowledge in the last decade. There is no doubt that smoking is a risk factor for the development of Graves' hyperthyroidism (GH) and even more so for Graves' ophthalmopathy (GO): all studies reach the same conclusion (1). The situation is less clear with respect to autoimmune hypothyroidism: some studies do report a higher prevalence of smokers presenting with Hashimoto's hypothyroidism (HH) whereas others are unable to find such an association $(1,2)$. Studies on smoking and autoimmune hypothyroidism are, however, few in number and heterogeneous in nature, and a recent meta-analysis failed to detect a significant association, although there was a trend (1).

New studies provide fascinating insights into the relationship between smoking and AITD. Unexpectedly, they raise the possibility that smoking may prevent the occurrence of $\mathrm{HH}$ (whereas it stimulates the development of GH). The studies also allow an evidence-based approach to determining whether cessation of smoking diminishes the risk of Graves' disease (GD) and whether passive smoking is relevant in this respect.

\section{Smoking protects against thyroid peroxidase antibodies}

There are now three studies that all imply that smoking may protect against the development of thyroid peroxidase antibodies (TPO-Ab). In 2003
Strieder and co-workers reported on risk factors for AITD in 759 euthyroid females with at least one relative with documented AITD (3). Current smoking (defined as smoking now, or having stopped smoking within 1 year of the study visit) was more prevalent in subjects without TPO-Ab $(<100 \mathrm{kU} / \mathrm{l})$ than in subjects with $\mathrm{TPO}-\mathrm{Ab} \geq 100 \mathrm{kU} / \mathrm{l}$ (38 vs $25 \%, P<0.001$ ). The frequency of ever smoking was similar between both groups ( 59 vs $52 \%$, NS). Current smoking was an independent determinant for the presence of TPO-Ab with an odds ratio of 0.688 (95\% CI $0.480-0.986)$. In 2004 Goh et al. likewise observed a lower prevalence of TPO-Ab in smokers than in non-smokers (52 vs $73 \%, P=0.06)$ among 102 patients with newly diagnosed GH (4).

The largest dataset comes from the NHANES III study, conducted between 1988 and 1994 and reflecting the entire non-institutionalised US population. After excluding subjects taking thyroid-altering medications from the 18148 persons who underwent thyroid testing, 15592 remaining subjects were analysed (5). Subjects were classified as smokers if serum cotinine was $>15 \mathrm{ng} / \mathrm{ml}$. Fewer smokers $(11 \%, 95 \%$ CI $10-19 \%)$ had thyroid antibodies (TPO-Ab $\geq 1.0 \mathrm{lU} / \mathrm{ml}$ ) than non-smokers (18\%, 95\% CI 17-19\%). The difference in prevalence of thyroid antibodies remained significant after adjustment for age, gender, race-ethnicity, and iodine status (Fig. 1). The relationship persisted upon analysing the association between smoking and the presence of TPO$\mathrm{Ab}$ (independent of thyroglobulin-antibody (Tg- $\mathrm{Ab}$ ) status), as was the case for $\mathrm{Tg}$ - $\mathrm{Ab}$ (independent of 


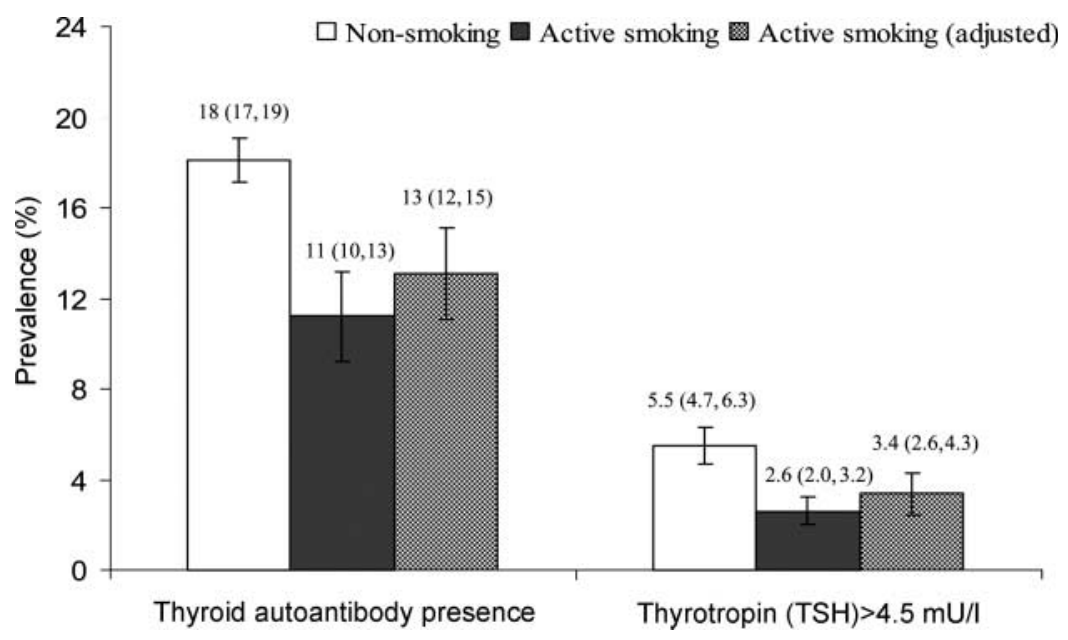

Figure 1 Prevalence of thyroid autoantibodies and elevated TSH concentrations among nonactive smokers and active smokers, excluding subjects taking thyroid-related medications. Adjustments include age, gender, race-ethnicity, and urinary iodine. Individuals with TSH levels below $0.1 \mathrm{mU} / \mathrm{l}$ were excluded from the analysis of TSH elevation. Lines represent 95\% Cls. From RM Belin et al. (5), with the permission of the Endocrine Society.
TPO-Ab status). The odds of having thyroid antibodies were lower by $1.1 \%$ for every $10 \mathrm{ng} / \mathrm{ml}$ increase in serum cotinine (OR 0.989, 95\% CI 0.983-0.995). There was a significant inverse linear relationship between log (cotinine) and $\log$ (TPO-Ab) levels, even after adjusting for cofounders. Smokers also less frequently had an elevated level of serum thyroid-stimulating hormone (TSH) of $>4.5 \mathrm{mU} / \mathrm{l}$ than non-smokers $(2.6 \%, 95 \% \mathrm{CI}$ $2.0-3.2$ vs $5.5 \%$, 95\% CI 4.7-6.3\%; Fig. 1). The OR for TSH elevation in smokers was 0.5 (95\% CI 0.4-0.6), and the odds were lower by $1.4 \%$ for every $10 \mathrm{ng} / \mathrm{ml}$ increase in serum cotinine. However, among persons without TSH elevation, smoke exposure was associated with $200 \%$ greater odds of having TSH levels of $0.1-0.4 \mathrm{mU} / \mathrm{l}$ (adjusted OR 2.0, 95\% CI 1.3-2.9), and every $10 \mathrm{ng} / \mathrm{ml}$ increase in cotinine was associated with $2 \%$ higher odds.

It can be concluded that smoking in a dosedependent manner is negatively associated with TPO$\mathrm{Ab}$ and elevated TSH, and positively with subnormal TSH. A probable explanation of these findings is that smoking inhibits the development of TPO-Ab and thereby protects, to a certain extent, against the occurrence of chronic lymphocytic thyroiditis and consequently against an elevated TSH. This line of reasoning is supported by a slightly higher serum TSH in TPO-Ab positive than in TPO-Ab negative healthy subjects with TSH values all within the normal reference range (3), and by a Danish population study indicating a lower prevalence of subclinical hypothyroidism among self-reported smokers with an adjusted OR of 0.47 (95\% CI 0.33-0.67) (6). In contrast, smoking appears to increase the risk of subnormal TSH values. Although the NHANES III study does not specify the cause of these subnormal TSH values, it might well be that these subjects are on their way to developing GH. Indeed, data from the large prospective Nurses Health Study II, with a 12-year follow-up, indicate cigarette smoking as a predictor of GH (7). The covariate-adjusted hazard ratio among current smokers was 1.93 (95\% CI 1.54-2.43).
The hazard ratio increased with the intensity of smoking and was 2.63 (95\% CI 1.71-4.04) among women smoking $\geq 25$ cigarettes daily.

AITD is thought to develop in relation to a particular genetic background and to be triggered by environmental factors. Based on recently obtained epidemiological data it could be speculated that the smoking behaviour of those individuals genetically susceptible to AITD determines, to some extent, whether they will develop $\mathrm{HH}$ or GH. In view of the manifold but poorly understood effects of smoking on the immune system, such a hypothesis might not be too speculative. It could be tested experimentally whether or not smoke exposure directs thyroid autoimmunity away from cell-mediated towards humoral immune reactions.

\section{Does cessation of smoking prevent AITD?}

According to the above-mentioned hypothesis, to stop smoking would decrease the risk of GH but increase the risk of HH. It is unknown whether ex-smokers put themselves at higher risk of autoimmune hypothyroidism by quitting smoking. In the NHANES III study there were no significant associations between a history of prior, but not current, smoking and any thyroid outcome, although there were fewer smokers among the 454 individuals taking thyroid-related medication $(16.6 \%, 95 \%$ CI $11.7-21.5)$ than among the individuals not reporting thyroid-altering medications $(32.2 \%$, 95\% CI 30.5-33.8) (5). Whereas hypothyroidism is easily treatable, GH and especially GO can be more difficult to treat. It follows that, particularly in view of the many other adverse health outcomes associated with smoking, the standard advice should always be to discontinue smoking. The question that then arises is whether there is good evidence that the risk of GD becomes lower in ex-smokers. Vestergaard's (1) metaanalysis indeed concludes that cessation of smoking lowers the risk of $\mathrm{GH}$ : the $\mathrm{OR}$ for current smoking 
(3.30, 95\% CI 2.09-5.22) is higher than for previous smokers $(1.41,95 \%$ CI $0.77-2.58, P=0.047)$. Even better evidence can be derived from the Nurses Health Study II, in which the covariate-adjusted hazard ratio among past smokers was 1.27 (95\% CI 1.03-1.56) vs 1.93 (95\% CI 1.54-2.43) among current smokers (7). The hazard ratios decreased with the number of years since a past smoker had stopped smoking, from 0.83 at 5 years to 0.52 at $>15$ years since quitting $(P=0.002$ for trend; hazard ratio for current smokers set at 1.00). It should be added that four observational studies have now been published, all indicating a higher recurrence rate of $\mathrm{GH}$ in smokers than in non-smokers following a course of antithyroid drugs. A Belgian study reported a relapse risk in patients without TSH receptor antibodies at the end of the drug course of $18 \%$ in non-smokers and of $57 \%$ in smokers; in the case of positive TSH receptor antibodies these figures were $86 \%$ in nonsmokers and $100 \%$ in smokers (8). Similar figures have been reported in a Norwegian study: after withdrawal of antithyroid drugs the relapse rate in smokers was higher than in non-smokers (58 vs $39 \%, P=0.009$ ) (9). A British study reported that smoking had a marginally significant $(P=0.081)$ deleterious effect on the likelihood of remission after antithyroid drug treatment for GD. The effect of smoking was, however, highly significant in males and indeed the deleterious effect on remissions may be restricted to males (OR 11.1, 95\% CI 1.25-98.5) (10). Finally, a German study has also indicated smoking as an independent risk factor for recurrent GH (11). Taken together, the available studies provide good evidence that cessation of smoking is beneficial in both primary and secondary prevention of $\mathrm{GH}$.

Smoking is a risk factor for $\mathrm{GH}$ but the odds for $\mathrm{GO}$ are much higher $(1.9,95 \%$ CI $1.1-2.7$ vs $7.7,95 \%$ CI $4.3-$ 13.7 in a case-control study on GH without and with eye changes respectively) (12); the odds increase progressively with more severe eye disease. Smoking has been identified as one of the risk factors for the development or worsening of eye changes after ${ }^{131} \mathrm{I}$ treatment of GH (13). The outcome of immunosuppressive treatment (glucocorticoids, retrobulbar irradiation) of GO is less favourable in smokers as compared to nonsmokers $(13,14)$. The data certainly suggest that refraining from smoking is useful in the secondary and tertiary prevention of GO; definite proof is lacking in this respect, however, because formal trials on smoking cessation in GO patients have not been undertaken. The best evidence for primary prevention of GO by quitting smoking comes from a study by Pfeilschifter and Ziegler (15). They report, among current smokers, a relative risk (RR) of diplopia of $1.8(95 \%$ CI $0.8-4.3)$ at $1-10$ cigarettes per day; the RR is 3.8 (95\% CI 1.9-9.7) for 11-20 cigarettes per day, and 7.0 (95\% CI 3.0-16.5) for $>20$ cigarettes per day, with similar figures for pre-optosis. Among ex-smokers who smoked $>20$ cigarettes per day, the RR for diplopia is 1.9 (95\% CI $0.5-7.7)$, and is no longer significant. As patients with GO are very much concerned (and rightly so) about their visual functions and appearance, the physician should grasp the opportunity to convince their patients to stop smoking. It is very likely to improve the outcome of the eye disease, and, because of their eye changes, patients might be more inclined than at other times to follow the advice to stop smoking.

\section{The effect of passive smoking}

There is concern about the effect of passive smoking, in this respect best illustrated by the financial support from the Flight Attendant Medical Research Institute for the NHANES III study at the time when smoking was still permitted in aircraft. It is, however, very difficult to evaluate the effect of passive smoking. The only available circumstantial evidence that passive smoking might be a risk factor for AITD is derived from a questionnaire study on childhood GO (16). Out of 1914 patients with childhood GH seen by respondents from 23 countries in the last 10 years, $576(30 \%)$ had GO. When grouped according to smoking prevalence among teenagers in the country of origin, it became evident that the proportion of GO patients among children with $\mathrm{GH}$ is highest in countries in which teenagers smoke most (Table 1). What is striking is that $52 \%$ of the children with GO in these countries (smoking prevalence $\geq 25 \%$ ) are 10 years old or younger, whereas the figure $(19 \%)$ is much lower in countries in which smoking prevalence among teenagers is less than $25 \%$. It is unlikely that children $\leq 10$ years of age smoke themselves; the high proportion of GO in this group is thus best explained by passive smoking as a result of

Table 1 Occurrence of childhood Graves' ophthalmopathy in Graves' hyperthyroidism as a function of smoking prevalence among teenagers in their country of origin.

\begin{tabular}{lcccc}
\hline & & & \multicolumn{2}{c}{ Graves' ophthalmopathy } \\
\cline { 3 - 5 } $\begin{array}{l}\text { Smoking prevalence } \\
\text { among teenagers }^{*}\end{array}$ & Graves' hyperthyroidism & Graves' ophthalmopathy & $\leq 10$ years (\%) & $11-18$ years (\%) \\
\hline$(1) \geq 25 \%$ & $644(100 \%)$ & $236(36.6 \%)$ & 52 & 48 \\
$(2) 20-25 \%$ & $818(100 \%)$ & $223(27.3 \%)$ & 15 & 85 \\
$(3)<20 \%$ & $452(100 \%)$ & $117(25.9 \%)$ & 24 & 76 \\
\hline
\end{tabular}

*Data per country based on WHO regional office for Europe, tobacco control database, 2003. Internet: http://data.euro.WHO.int/tobacco. 
living in an environment in which $25 \%$ or more of their peers smoke.

It is of interest that, based on the WHO regional office for Europe, tobacco control database, 2003, all the countries $(100 \%)$ that are included in the first group have a smoking prevalence higher than 25\% among adults, while only $50 \%$ and $40 \%$ of the countries in the second and third groups exhibit such a prevalence.

In conclusion, new studies have shown that smoking may protect against the development of TPO-Ab, which may result in a decreasing risk of $\mathrm{HH}$, whereas it stimulates the development of $\mathrm{GH}$. According to the above-mentioned hypothesis, to stop smoking would decrease the risk of $\mathrm{GH}$ but increase the risk of $\mathrm{HH}$. Also, smoking has been identified as one of the risk factors for the development or worsening of eye changes after ${ }^{131} \mathrm{I}$ treatment of GH. Additionally, the outcome of medical treatment of GO is less favourable in smokers than nonsmokers. There is concern also about the effect of passive smoking on AITD. In a recent study it has been shown that the latter may have a deleterious effect on childhood GO.

\section{References}

1 Vestergaard P. Smoking and thyroid disorders - a meta-analysis. European Journal of Endocrinology 2002146 153-161.

2 Vestergaard P, Rejnmark L, Weeke J, Hoeck HC, Nielsen HK, Rungby J, Laurberg P \& Mosekilde L. Smoking as a risk factor for Graves' disease, toxic nodular goiter, and autoimmune hypothyroidism. Thyroid 200212 69-75.

3 Strieder TGA, Prummel MF, Tijssen JGP, Endert E \& Wiersinga WM. Risk factors and prevalence of thyroid disorders in a cross-sectional study among healthy female relatives of patients with autoimmune thyroid disease. Clinical Endocrinology (Oxford) 200359 396-401.

4 Goh SY, Ho SC, Seah LL, Fong KS \& Khoo DHC. Thyroid autoantibody profiles in ophthalmic dominant and thyroid dominant Graves' disease differ and suggest ophthalmopathy is a multiantigenic disease. Clinical Endocrinology (Oxford) 200460 600-607.

5 Belin RM, Astor BC, Powe NR \& Ladenson PW. Smoke exposure is associated with a lower prevalence of serum thyroid autoantibodies and thyrotropin concentration elevation and a higher prevalence of mild thyrotropin concentration suppression in the third National Health and Nutrition Examination Survey (NHANES III). Journal of Clinical Endocrinology and Metabolism $2004896077-6086$.

6 Knudsen N, Bulow I, Laurberg P, Perrild H, Ovesen L \& Jorgensen T. High occurrence of thyroid multinodularity and low occurrence of subclinical hypothyroidism among tobacco smokers in a large population study. Journal of Endocrinology 2002175 571-576.

7 Holm IA, Manson JE, Michels KB, Alexander EK, Willett WC \& Utiger RD. Smoking and other lifestyle factors and the risk of Graves' hyperthyroidism. Archives of Internal Medicine 2005165 $1606-1611$.

8 Glinoer D, de Nayer P \& Bex M, Belgian Collaborative Study Group on Graves' Disease. Effects of l-thyroxine administration, TSHreceptor antibodies and smoking on the risk of recurrence in Graves' hyperthyroidism treated with antithyroid drugs: a doubleblind prospective randomized study. European Journal of Endocrinology $2001 \mathbf{1 4 4} 475-483$.

9 Nedrebo BG, Holm PI, Uhlving S, Sorheim JI, Skeie S, Eide GE, Husebye ES, Lien EA \& Aanderud S. Predictors of outcome and comparison of different drug regimens for the prevention of relapse in patients with Graves' disease. European Journal of Endocrinology $2002147583-589$.

10 Kimball LE, Kulinskaya E, Brown B, Johnston C \& Farid NR. Does smoking increase relapse rates in Graves' disease? Journal of Endocrinological Investigation 200225 152-157.

11 Hoermann R, Quadbeck B, Roggenbuck U, Szabolcs I, Pfeilschifter J, Meng W, Reschke K, Hackenberg K, Dettmann J, Prehn B, Hirche H \& Mann K, Basedow Study Group. Relapse of Graves' disease after successful outcome of antithyroid drug therapy: results of a prospective randomized study on the use of levothyroxine. Thyroid $2002121119-1128$.

12 Prummel MF \& Wiersinga WM. Smoking and risk of Graves' disease. Journal of the American Medical Association 1993269 479-482.

13 Bartalena L, Marcocci C, Tanda ML, Manetti L, Dell'Unto E, Bartolomei MP, Nardi M, Martino E \& Pinchera A. Cigarette smoking and treatment outcomes in Graves ophthalmopathy. Annals of Internal Medicine 1998129 632-635.

14 Eckstein A, Quadbeck B, Mueller G, Rettenmeier AW, Hoermann R, Mann K, Steuhl P \& Esser J. Impact of smoking on the response to treatment of thyroid associated ophthalmopathy. British Journal of Ophthalmology $2003 \mathbf{8 7} 773-776$.

15 Pfeilschifter J \& Ziegler R. Smoking and endocrine ophthalmopathy: impact of smoking severity and current vs lifetime cigarette consumption. Clinical Endocrinology (Oxford) 199645 477-481.

16 Krassas GE, Segni M \& Wiersinga WM. Childhood Graves' ophthalmopathy: results of a European questionnaire study. European Journal of Endocrinology 2005153 515-521.

Received 17 January 2006

Accepted 3 March 2006 